\title{
sciendo
}

\section{Trends of Goal Scoring Patterns in Soccer: A Retrospective Analysis of Five Successive FIFA World Cup Tournaments}

\author{
by \\ Alliance Kubayi ${ }^{1}$, Abel Toriola ${ }^{1}$
}

\begin{abstract}
This study analysed the 795 goals scored during a total of 320 matches played in five successive FIFA World Cup tournaments (1998-2014). Data were obtained through YouTube videos and analysed by means of Longomatch software. The variables analysed included the number of goals scored per half (45-min period), per 15-min period, and per 30-min period of extra time, goal scoring zones, goals scored by substitutes, types of goals scored, and goals scored according to the playing position. With regard to 15-min period analysis, most goals were scored between the 76th and 90th minutes (24.7\%) of the game in all five World Cup competitions. Chi-square analyses showed no significant ( $p>$ 0.05) differences in the frequency of goal scoring patterns per 45-min and 15-min periods in the five World Cup tournaments. Most goals were scored from inside the goal (23.8\%) and penalty (14.6\%) areas. The greatest number of goals was scored by strikers (54.2\%), followed by midfielders (33.3\%) and defenders (2.3\%). These findings provide practical implications for improving goal-scoring performance in soccer.
\end{abstract}

Key words: soccer, tactics, video analysis, goals, performance.

\section{Introduction}

Soccer is undoubtedly the world's most popular sport, played by more than 240 million people as members of 1.4 million teams (Acar et al., 2009). It was reported that the 2014 FIFA World Cup in Brazil attracted a global television audience of 3.2 billion people, with more than one billion people watching the final (FIFA, 2015). Of course, the soccer World Cup provides an opportunity to observe the best teams and players in the world. In addition to the anthropometric, psychological, and physiological research, performance analysis among soccer players in terms of techniques and tactics has become popular (Acar et al., 2009; Castellano et al., 2012; Clemente et al., 2015; Moura, et al., 2015; Smpokos et al. 2018).

Regardless of the popularity associated with the sport, a low number of goals scored during a match is one of the features of modern soccer (Phukan et al., 2015; Kalinowski et al., 2019). This trend is striking considering the fact that goal scoring provides the most exciting and fascinating positive experience for soccer fans as well as coaches and analysts. Therefore, it is essential that soccer coaches and technical staff pay more attention to goal scoring patterns as such information can be helpful in devising strategies that could maximise team success. Despite a plethora of studies on the analysis of goals scored in soccer (e.g., Acar et al., 2009; Njororai, 2013; Phukan et al., 2015; Yiannakos and Armatas, 2006), most previous research has been limited to one tournament, either at the national or international level. Indeed, there is a dearth of research concerning the goal scoring trends in the FIFA World Cup.

To date, only one study could be found, which was conducted by Armatas et al. (2007) and focussed on the relationship between time and goal scoring in the 1998, 2002, and 2006 FIFA World Cups. The results indicated that most goals were scored in the second half of the game in all three World Cup tournaments. With regard to 15-min analysis, it was found that most goals were scored between the $76^{\text {th }}$ and $90^{\text {th }}$ min of the game, and the highest number of goals was recorded towards the

1 - Department of Sport, Rehabilitation and Dental Sciences, Tshwane University of Technology, South Africa.

Authors submitted their contribution to the article to the editorial board.

Accepted for printing in the Journal of Human Kinetics vol. 69/2019 in September 2019. 
end of matches (Armatas et al., 2007). Although the study by Armatas et al. (2007) has provided baseline information with reference to the goal scoring patterns from an international perspective, it is not without limitations, with the most notable being the restriction to goal scoring frequency (i.e., goals scored per 45-min and 15-min periods). As a result, their study did not provide a complete picture of the goal scoring patterns at FIFA World Cup competitions.

Therefore, this study seeks to retrospectively analyse the goal scoring patterns and trends in the last five successive FIFA World Cup tournaments (1998-2014). Specifically, the objectives of the study were to examine the frequency of goals scored, goal scoring zones, the number of goals scored by substitutes, types of goals scored (set play or open play), and goals scored according to the playing position. As Abt et al. (2002) argued, analysis of goal scoring patterns could provide helpful information to both coaches and sports scientists, as the relationship between goal scoring and time would appear to be linked to those aspects of play which inherently change as a match progresses, such as physical conditioning and tactical play.

\section{Methods}

Following institutional ethics approval, 64 matches from each of the five successive FIFA World Cups (1998 - France, 2002 - Korea/Japan, 2006 - Germany, 2010 - South Africa, and 2014 Brazil) were studied ( $\mathrm{N}=320$ matches). The FIFA World Cup tournament was selected because it involved the participation of top international teams from all over the world (Armatas et al., 2007). Data were obtained through YouTube videos, which was a similar data collection procedure to that used in previous research on soccer (Furley et al., 2017). The matches were analysed by the principal researcher using Longomatch software in which all actions were recorded on an electronic sheet.

The variables analysed included the frequency of goals scored per half (first half, second half, extra time), 15-min period (1-15, 1630, 31-45, 45-60, 61-75, 76-90), and 30-min period of extra time (91-105, 105-120), goal scoring zones (penalties, inside the goal area, inside the penalty area, outside the penalty area), the number of goals scored by substitutes, types of goals scored (open play or set play), and the number of goals scored according to the playing position (defender, midfielder, striker). For ease of analysis, all the goals scored at the five successive FIFA World Cup tournaments were tallied and combined. Thereafter, all variables were entered into a Microsoft Excel ${ }^{\circledR}$ spreadsheet, imported into Statistical Package for Social Sciences (SPSS) software and analysed descriptively (frequencies and percentages). A Chi-square test was used to examine significant differences in the frequency of goals scored. The level of significance was set at $p$ $\leq 0.05$.

\section{Results}

A total of 795 goals were scored in 320 matches played in the last five successive FIFA World Cups. Most goals were scored at the 1998 (an average of 2.67 per match) and 2014 (an average of 2.67 per match) World Cup tournaments, while the least were recorded during the 2006 (an average of 2.30 per match) and 2010 (an average of 2.27 per match) competitions (Table 1).

With regard to 15-min period analysis, most goals were scored during the $76^{\text {th }}-90^{\text {th }}$ min $(24.7 \%)$ period of the game in all five World Cups. The lowest number of goals was observed between the $16^{\text {th }}$ and $30^{\text {th }}$ minutes of the matches in the 1998 $(11.1 \%)$ and $2002(11.8 \%)$ tournaments, and also in the first 15 minutes of the matches in the 2006 $(15.6 \%), \quad 2010 \quad(9.7 \%), \quad$ and $2014 \quad(10.5 \%)$ tournaments. Chi-square analyses showed no significant $(p>0.05)$ differences in the frequency of goal scoring patterns per 15-min period in the five World Cups. Despite this non-significant difference, the frequency of goal scoring was time dependent, and goals were scored as time progressed (Figure 1).

Table 2 shows zones from which goals were scored during the World Cup events. Overall, in all the competitions, few goals were scored from penalties $(8.0 \%)$ and from outside the penalty area $(14.6 \%)$, while most goals were scored from inside the goal $(23.8 \%)$ and penalty $(53.6 \%)$ areas.

Figure 2 illustrates the patterns of goals scored by substitutes in the five World Cup competitions. The results showed that substitutes scored more goals in 2006 (15.6\%) and 2014 (18.7\%) than in 1998 (8.8\%) and 2002 (13.0\%).

Figure 3 illustrates the types of goals 
scored. Overall, most goals were scored from open play, with the highest number of goals recorded in 2010 (75.9\%) and 2014 (77.8\%). The 1998 (63.7\%) and 2006 (68.7\%) World Cup competitions had the least goals from open play.

Most set play goals were scored in 1998 (36.3\%) and $2006(31.3 \%)$, declining to $24.1 \%$ and $22.2 \%$ in
2010 and 2014, respectively.

Table 3 presents the distribution of goals according to the player position. As expected, most goals were scored by strikers (54.2\%), followed by midfielders $(33.3 \%)$ and defenders $(10.2 \%)$, in all the World Cups.

Table 1

Goal scoring frequency (FIFA World Cup tournaments: 1998-2014)

\begin{tabular}{|c|c|c|c|c|c|c|}
\hline Time interval & 1998 & 2002 & 2006 & 2010 & 2014 & Total \\
\hline 01-15 & $25 \pm 14.6$ & $25 \pm 15.5$ & $23 \pm 15.6$ & $14 \pm 9.7$ & $18 \pm 10.5$ & $105 \pm 13.2$ \\
\hline $16-30$ & $19 \pm 11.1$ & $19 \pm 11.8$ & $24 \pm 16.3$ & $23 \pm 15.9$ & $25 \pm 14.6$ & $110 \pm 13.8$ \\
\hline $31-45$ & $26 \pm 15.2$ & $25 \pm 15.5$ & $23 \pm 15.6$ & $22 \pm 15.2$ & $22 \pm 12.9$ & $118 \pm 14.8$ \\
\hline $45-60$ & $31 \pm 18.1$ & $28 \pm 17.4$ & $19 \pm 12.9$ & $22 \pm 15.2$ & $24 \pm 14.0$ & $124 \pm 15.6$ \\
\hline $61-75$ & $24 \pm 14.0$ & $30 \pm 18.6$ & $11 \pm 7.5$ & $27 \pm 18.6$ & $33 \pm 19.3$ & $125 \pm 15.7$ \\
\hline $76-90$ & $45 \pm 26.3$ & $31 \pm 19.3$ & $44 \pm 29.9$ & $35 \pm 24.1$ & $41 \pm 24.0$ & $196 \pm 24.7$ \\
\hline \multicolumn{7}{|l|}{ Extra time } \\
\hline 91-105 & $00 \pm 0.0$ & $02 \pm 1.2$ & $01 \pm 0.7$ & $01 \pm 0.6$ & $03 \pm 1.8$ & $07 \pm 0.9$ \\
\hline $105-120$ & $01 \pm 0.6$ & $01 \pm 0.6$ & $02 \pm 1.4$ & $01 \pm 0.6$ & $05 \pm 2.9$ & $10 \pm 1.3$ \\
\hline Total & $171 \pm 100$ & $161 \pm 100$ & $147 \pm 100$ & $145 \pm 100$ & $171 \pm 100$ & $795 \pm 100$ \\
\hline $\begin{array}{l}\text { Number of } \\
\text { games played }\end{array}$ & 64 & 64 & 64 & 64 & 64 & 320 \\
\hline Average & 2.67 & 2.52 & 2.30 & 2.27 & 2.67 & 2.48 \\
\hline
\end{tabular}

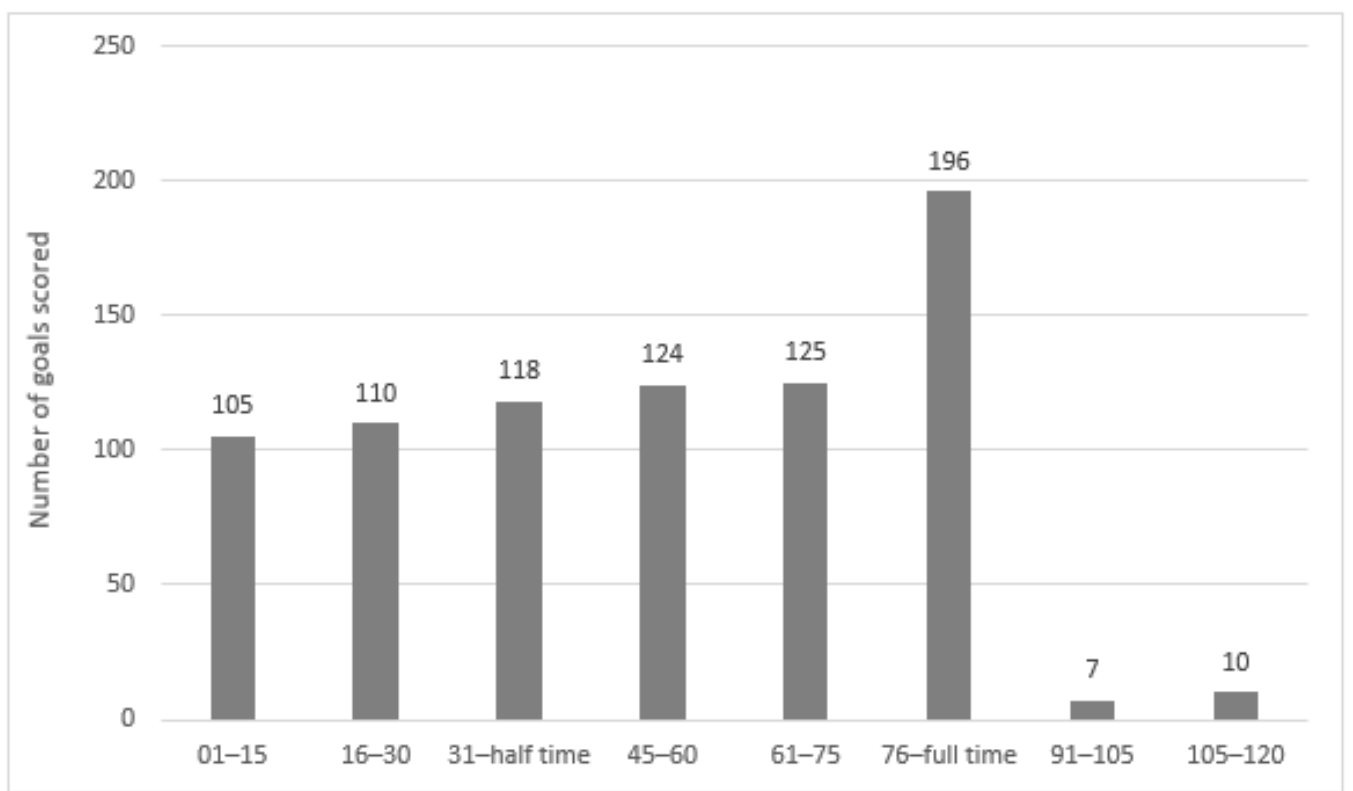

Figure 1

Goal scoring distribution (FIFA World Cup tournaments: 1998-2014) 


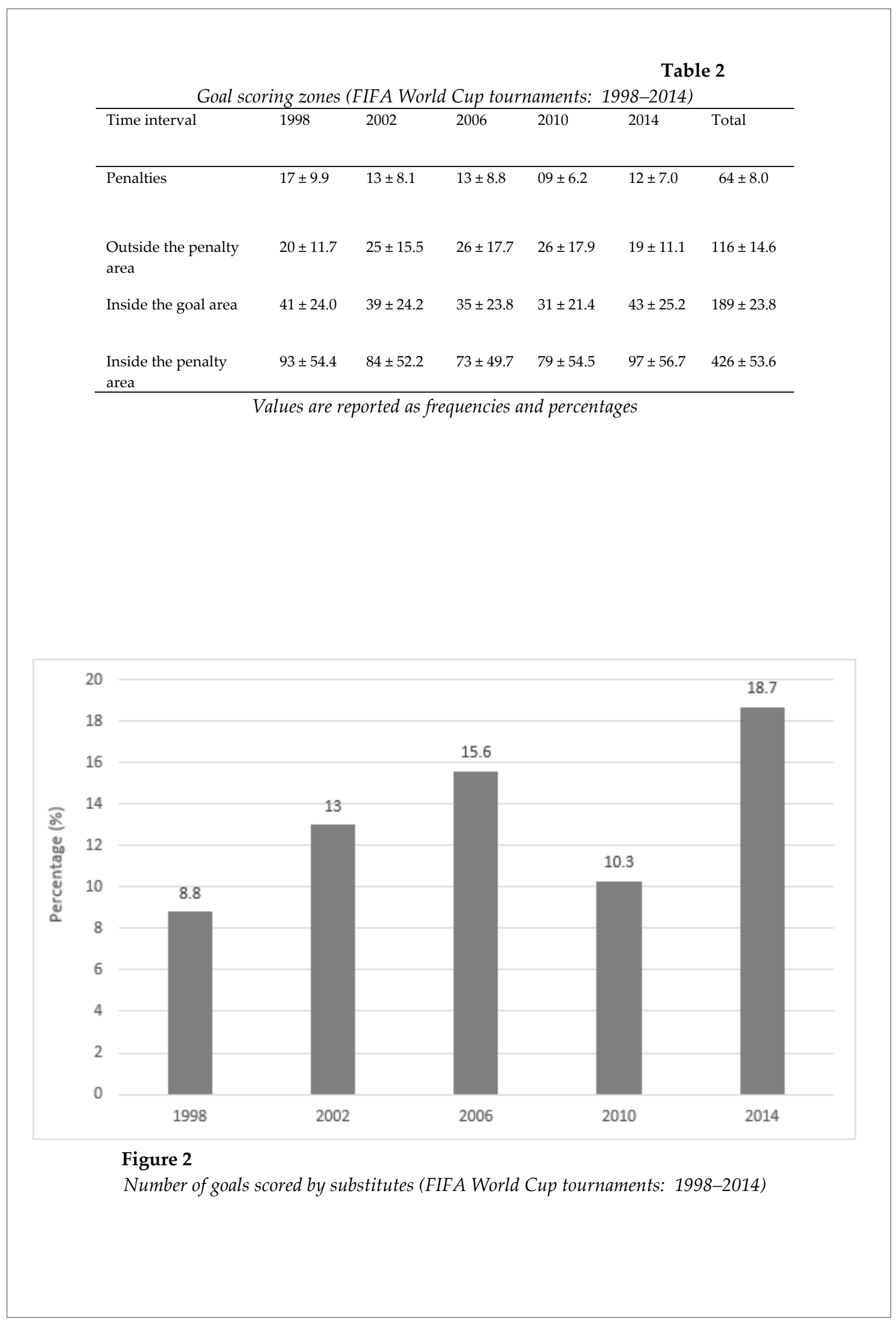




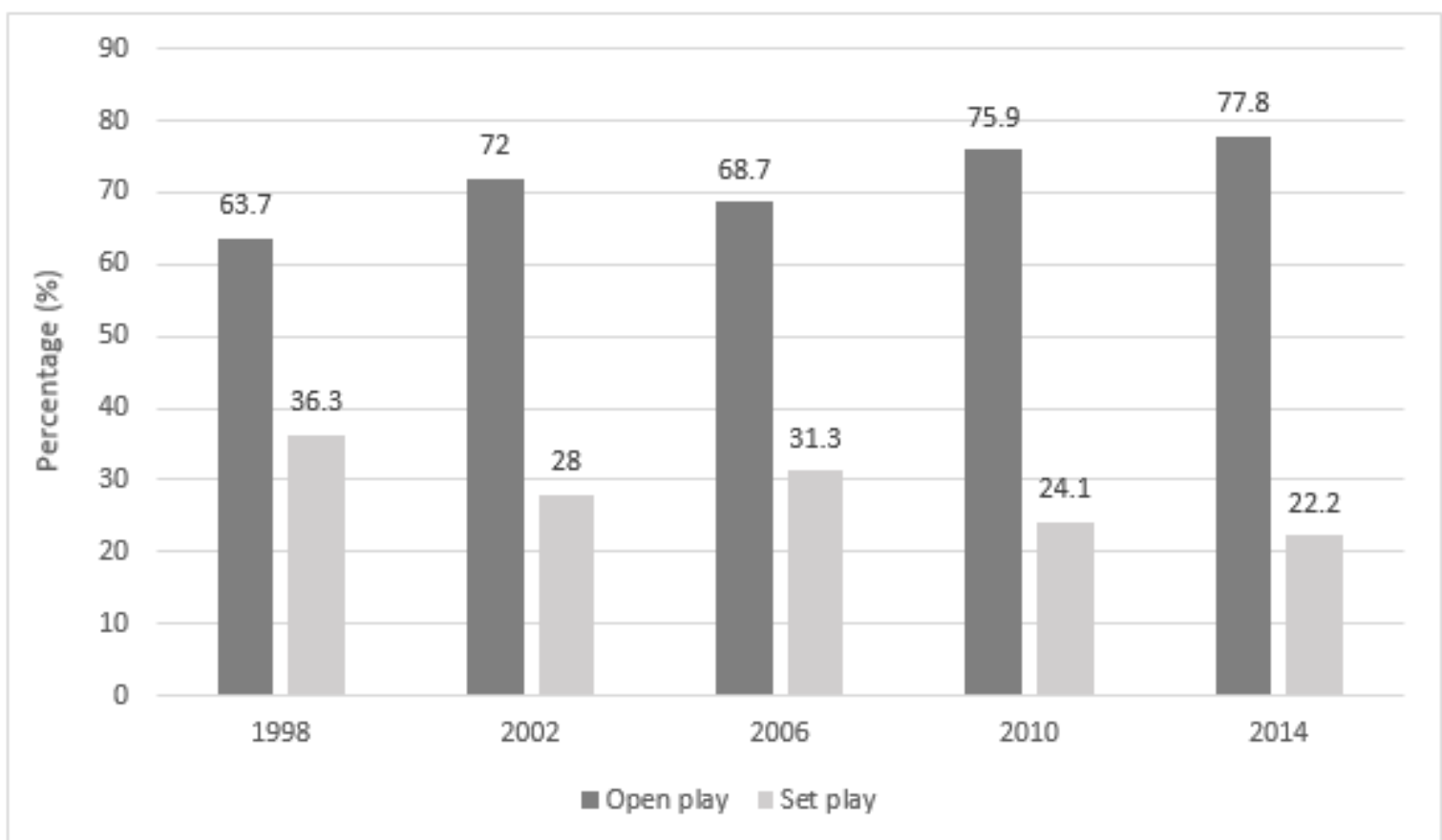

Figure 3

Types of goals scored (FIFA World Cup tournaments: 1998-2014)

Table 3

Analyses of goal scoring according to the player position (FIFA World Cup tournaments:

\begin{tabular}{|c|c|c|c|c|c|c|}
\hline & 1998 & 2002 & 2006 & 2010 & 2014 & Total \\
\hline Strikers & $93 \pm 54.4$ & $88 \pm 54.7$ & $79 \pm 53.7$ & $77 \pm 53.1$ & $94 \pm 55.0$ & $431 \pm 54.2$ \\
\hline Midfielder & $59 \pm 34.5$ & $53 \pm 32.9$ & $47 \pm 32.0$ & $50 \pm 34.5$ & $56 \pm 32.7$ & $265 \pm 33.3$ \\
\hline Defenders & $15 \pm 8.8$ & $17 \pm 10.5$ & $17 \pm 11.6$ & $16 \pm 11.0$ & $16 \pm 9.4$ & $81 \pm 10.2$ \\
\hline Own goal & $04 \pm 2.3$ & $03 \pm 1.9$ & $04 \pm 2.7$ & $02 \pm 1.4$ & $05 \pm 2.9$ & $18 \pm 2.3$ \\
\hline
\end{tabular}

Values are reported as frequencies and percentages 


\section{Discussion}

The aim of this study was to retrospectively analyse the trends of goal scoring patterns in five successive FIFA World Cup tournaments. Overall, this study found that the goal scoring patterns were time dependent. It was also noted that the number of goals scored increased as a game progressed, with the highest proportion of goals recorded in the last 15 minutes of the match. Given that the sample of this study comprised five World Cup competitions, the present findings support those of previous research (Abt et al., 2002), which reported an upward trend in the goal scoring frequency over the course of the game. Most goals being scored in the final 15-min period of the game could be attributed to factors such as differences in conditioning levels and lapses of concentration among players (Acar et al., 2009). This phenomenon could be explained by player fatigue towards the end of the game. Players who have better physical conditioning are able to minimise the effects of fatigue, especially towards the end of the game, when they tend to gain an advantage over their opponents with inferior conditioning (Abt et al., 2002; Carling et al., 2005).

Similarly, conceding goals towards the end of the game could be associated with 'mental fatigue' among players as a result of continued physical effort, which could lead to tactical mistakes and unforced errors that open up goal scoring opportunities (Carling et al., 2005). The late surge in goal scoring may also be attributed to players' time consciousness and urgency in spite of the decline in physical capabilities. Carling et al. (2005) further indicated that due to the fact that critical incidents in contesting ball possession usually occurred in the final 15 minutes of the game, goal scoring chances were more likely to increase. Despite the occurrence of fatigue, a team that is physically, mentally, tactically, and technically prepared to endure the entire 90 minutes of intense match play is more likely to be an effective and cohesive unit (Carling et al., 2005).

Strategies such as ensuring adequate hydration prior to and during the match, as well as dietary manipulation, can delay the effects of fatigue (Abt et al., 2002). Carling et al. (2005) reiterated that players with sound nutritional preparation for matches tended to show a more stable work-rate profile throughout the game than those who prepared casually. Additionally, the manner in which substitutes are utilised by the coach plays an important role in preventing the occurrence of fatigue towards the end of the game (Abt et al., 2002). The present study showed that substitute players scored most goals during the 2014 FIFA World Cup. The overall work rate of substitutes could also assist fatigued players. For example, in their study, Mohr et al. (2003) found that 13 substitutes covered $25 \%$ more distance during the last quarter of the game, engaging in more high-intensity running than the other players.

Results of the present study further showed that most goals were scored from open play, with a noticeable increase between the 2006 and 2014 World Cup competitions. In contrast, the number of set play goals decreased considerably between the 2006 and 2014 World Cups. Most goals were scored from inside the penalty area. A plausible reason for this finding could be that it is easier to score goals from this location (Yanci, 2015). Another possible explanation could be that the players prefer to be near the goalpost, but not in the goalkeeper's range so that they can shoot the ball with less distraction (Muhammd et al., 2013). The fact that fewer goals were scored from outside the penalty area may be ascribed to high-quality goalkeeping and compact defence structures. Similarly, the relatively few goals scored from outside the penalty area may be a reflection of the teams' ability to defend efficiently and effectively in their own areas (UEFA, 2016). It is not surprising in the present study that most goals were scored by strikers. However, the efficiency of defenders and midfielders in moving forward close to the goal area puts pressure on the opposition team and increases chances of goal scoring (Acar et al., 2009).

\section{Conclusion}

The results of this study showed that a total of 795 goals were scored during 320 games, with most goals recorded in the 1998 and 2014 FIFA World Cup tournaments. Concerning the 15min analysis, most goals were recorded between the $76^{\text {th }}$ and $90^{\text {th }}$ minutes in all five World Cup competitions. It was further found that the highest proportion of goals emerged from inside the goal and penalty areas. The results also showed that most goals were scored by strikers, followed by midfielders and defenders, in all the World Cup 
tournaments. Soccer coaches should take note of the findings of the present study in order to increase goal-scoring opportunities. Future research should focus on investigating the offensive tactics of goal scoring patterns in soccer.

\section{Practical implications}

Based on the findings of this study, several recommendations can be made. First, this study suggests that soccer coaches should ensure that players are given sufficient physical conditioning to endure the full $90+$ minutes of the game. Players who are physically prepared are likely to have higher levels of concentration, which will minimise errors such as poor marking, committing fouls, and so forth. Second, strategies such as dietary manipulation and adequate hydration before and during matches should be applied, as they may delay the onset of fatigue throughout the game. Finally, it should be noted that match analysis should not be regarded as an end in itself, as the outcome of a soccer match is determined by multidimensional factors such as fitness and psychological preparation, among others. However, it is important for soccer coaches to include the results of match analyses as part of their training programme in order to maximise the overall success of their teams.

\section{References}

Abt GA, Dickson G, Mummery WK. Goal scoring patterns over the course of a match: An analysis of the Australian National Soccer League. In Spinks W, Reilly T, Murphy T (Eds.), Science and Football IV (pp. 107-111). London: Routledge; 2002

Acar MF, Yapicioglu B, Arikan N, Yalcin S, Ates N, Ergun M. Analysis of goals scored in the 2006 World Cup. In Reilly T, Korkusuz F (Eds.), In Science and Football VI (pp. 235-242). London: Routledge; 2009

Armatas V, Yiannakos A, Sileloglou P. Relationship between time and goal scoring in soccer games: analysis of three World Cups. Int J Perf Anal Sport, 2007; 7: 48-58

Carling C, Williams A, Reilly T. The handbook of soccer match analysis: A systematic approach to improving performance. London: Routledge; 2005

Castellano J, Casamichana D, Lago-Penas C. The use of match statistics that discriminate between successful and unsuccessful soccer teams. J Hum Kinet, 2012; 31: 139-147

Clemente FM, Couceiro MS, Martins FML, Mendes RS. Using network metrics in soccer: a macro-analysis. J Hum Kinet, 2015; 45: 123-134

FIFA. 2014 FIFA World Cup ${ }^{\mathrm{TM}}$ reached 3.2 billion viewers, one billion watched final-Recent findings, 2015. Available at http://www.fifa.com/worldcup/news/y=2015/m=12/news. Accessed on 20.06.2017

Furley P, Noël B, Memmert D. Attention towards the goalkeeper and distraction during penalty shootouts in association football: a retrospective analysis of penalty shootouts from 1984 to 2012. J Sport Sci, 2017; 35: $873-879$

Kalinowski P, Bojkowski Ł, Śliwowski R. Motor and psychological predispositions for playing football. TRENDS Sport Sci, 2019; 2(26): 51-56

Mohr M, Krustrup P, Bangsbo J. Match performance of high-standard soccer players with special reference to development of fatigue. J Sport Sci, 2003; 21: 519-528

Moura FA, Santana JE, Vieira NA, Santiago PRP, Cunha SA. Analysis of soccer players' positional variability during the 2012 UEFA European Championship: A case study. J Hum Kinet, 2015; 47(1): 225-236

Muhammd S, Norasrudin S, Rahmat A. Differences in goal scoring and passing sequences between winning and losing team in UEFA-EURO Championship 2012. Int J Soc Behav Educ Eco Managet Eng, 2013; 7: 224-229

Njororai WWS. Analysis of goals scored in the 2010 World Cup soccer tournament held in South Africa. J Phys Educ Sport, 2013; 13: 6-13 
Phukan S, Singh OJ, Thapa S. Analysis of goal scoring pattern at Inter College Football Tournament. Int J Appl Res, 2015; 1: 219-220

Smpokos E, Mourikis Ch, Linardakis M. Seasonal changes of physical (motor) activities in professional Greek football players. TRENDS Sport Sci, 2018; 2(25): 99-107

UEFA. How the 108 goals at UEFA EURO 2016 went in-Recent findings 2016. Available at http://www.uefa.com/uefaeuro/news/newsid=2387449.html; Accessed 21.05.2017

Yanci J. Analysis of goals scored by players with cerebral palsy in official football 7-a-side matches. Kinesiol, 2015; 47: 202-207

Yiannakos A, Armatas V. Evaluation of the goal scoring patterns in European Championship in Portugal 2004. Int J Perf Anal Sport, 2006; 6: 178-188

\section{Corresponding author:}

\section{Alliance Kubayi}

Tshwane University of Technology

Department of Sport, Rehabilitation and Dental Sciences

Republic of South Africa

Pretoria 0001

Phone number: +27 123824272

Fax number: +27 123825801

E-mail: kubayina@tut.ac.za 